\title{
Postoperative Outcome of Duodenal Ulcer (DU) Perforation in Rajshahi Medical College Hospital: A Study of 300 Cases
}

Dr. Mohibul Hassan ${ }^{1}$, Dr. Subal Chandra Paul ${ }^{2 *}$, Dr. Sanchita Sarker ${ }^{3}$

${ }^{1}$ Department of Surgery, Rajshahi Medical College Hospital, Bangladesh

${ }^{2}$ Department of Surgery, Rajshahi Medical College Hospital, Bangladesh

${ }^{3}$ Department of Pediatrics, Rajshahi Medical College Hospital, Bangladesh

DOI: $10.36347 /$ sjams.2021.v09i01.017

| Received: 28.12.2020 | Accepted: 12.01.2021 | Published: 16.01.2021

*Corresponding author: Dr. Subal Chandra Paul

\section{Abstract}

Original Research Article

Introduction: Peptic Ulcer Disease (PUD) is one of the most common causes of acute abdomen in Bangladesh. Aim of the study: The aim of the study is to find out the post-operative outcome (Mortality and Morbidity) and to correlate the outcome with the prognostic factors. Method: This study was carried out in the Department of surgery, Rajshahi Medical college Hospital, Rajshahi, Bangladesh during the period from Jan 2015 to Dec 2019 for five years with the aim to find out the surgical outcome and associated prognostic factors. In this study 300 cases of perforated duodenal ulcer were randomly selected. Both male and female patients of any age group diagnosed as duodenal ulcer perforation were included in this study but the patients having gastric ulcer perforation were excluded because the surgical modality and outcome variables of those patients are different. The diagnosis of peptic ulcer perforation was made by the history of sudden onset of severe abdominal pain with clinical signs of diffuse peritonitis and presence of radiological sign of pneumoperitoneum. Per operative finding of gastric ulcer perforation or any suspicion of malignancy were excluded from the study. Any patients with traumatic pneumoperitonium were also excluded from the study. Results: Among the 300 cases, most of the patients were male $(91 \%=273)$, from low socioeconomic family $(77 \%=231)$ and from rural areas $(81 \%=243)$. Many patients arrived at hospital with significant lapse of time of average 2 days delay ranging from 1 day to 9 days and many patients had significant hemodynamic and biochemical abnormalities with or without systemic sepsis and septicemia. A number of patients were presented with single or multiple co morbidities like DM, IHD, HTN, COPD etc. Among the 300 cases, 14 (4.7\%) mortality was found, Wound infection (SSI) rate was $13.5 \%$ (41), Burst abdomen 08 (3\%) and Chest infection was $3110 \%$ in the form of pulmonary atelectasis or pneumonia. Conclusion: Mortality was found to have related with old age with limited physiological reserves due to multiple co morbidities, presence of profound shock and dehydration, presence of sepsis or septicemia and delay more than three days lapsed to reach in the hospital. Other post operative morbidities like wound infection (SSI) in the form of superficial, deep and organ space type along with the chest complications in the form of atelectasis and pneumonia were also studied here which were significantly related with the treatment cost and prolonged hospital stay.

Keywords: Postoperative Outcome, Duodenal Ulcer (DU) Perforation, Hospital stay.

Copyright $\odot$ (C) 2021 The Author(s): This is an open-access article distributed under the terms of the Creative Commons Attribution 4.0 International License (CC BY-NC 4.0) which permits unrestricted use, distribution, and reproduction in any medium for non-commercial use provided the original author and source are credited.

\section{INTRODUCTION}

Perforated gastric and duodenal ulcer is a common surgical emergency worldwide which is associated with high morbidity and mortality. Peptic ulcer disease (PUD) affects 4 million people around the world yearly out of which $10 \%-20 \%$ of these patients develop complications and $2 \%-14 \%$ of the ulcers perforate. Perforated peptic ulcer (PPU) is a life threatening disease and the mortality varies from $10 \%$ $40 \%$ [1]. Moller et al shoed that, Mortality and morbidity following perforated peptic ulcer (PPU) is substantial, and mortality rates of $25-30 \%$ have been reported in different published studies [2, 3]. Globally the incidence of peptic ulcer disease is said to have fallen in recent years. Also recent advances have taken place in both diagnosis and management of peptic ulcer disease, namely improvements in endoscopic diagnostic and therapeutic facilities, the increased use of proton pump inhibitors and Helicobacter pylori eradication therapies. In spite of all these, peptic ulcer perforation rates have remained unchanged [4] and therefore remain a major health challenge. Elective peptic ulcer surgery 
was common before the discovery that peptic ulcer is caused by $\mathrm{H}$. pylori and non-steroidal anti-inflammatory medications (NSAIDs) [5]. The discovery changed the management of the disease but still its complication could be seen as an emergency in the form of perforation or bleeding and stenosis [6]. A perforated ulcer can present early with chemical peritonitis or late with septic peritonitis. In late stage, it could cause severe sepsis which might lead to organ failure and mortality. Emergency surgical treatment is recommended for this condition [7]. The management trend of peptic ulcer disease has become changed over the last three decades, possibly because of the introduction of triple therapy management for these patients; nevertheless, the patients could still present with complications like perforation, bleeding or stenosis [8]. Surgery is most successful mode of treatment of perforated peptic ulcer peritonitis. Formal acid-reducing procedures like vagotomy with or without drainage procedures, which has historically been the mainstay of PPU therapy, are now being replaced by simpler procedures, such as omental patch primary closure of the perforation owing to better understanding of the pathophysiology of peptic ulcer and successful eradication of $\mathrm{H}$. pylori treatment. Perforated duodenal ulcer treated by patch repair with a vascularized omental pedicle commonly referred to as a Graham patch or omentopexy. However, in cases of perforated gastric ulcer, either ulcer excision or repair of the defect or biopsy and omental patch is the most expeditious approach in the emergency setting [9]. Poor outcomes in PPU have been associated with increasing age, major medical illness, peri-operative hypotension and delay in diagnosis and management [10]. Surgical repair should not be delayed in patients with general peritonitis because every hour of delay increases the mortality risk [11]. Peptic ulcer disease is one of the most prevalent diseases of the gastrointestinal tract. The common complications of peptic ulcer disease are bleeding, perforation and obstruction. Perforation remains a major life threatening complication. Duodenal, antral and gastric body ulcers account for $60 \%, 20 \%$ and $20 \%$ ulcers among the peptic ulcer perforations respectively. The current treatment of perforated peptic ulcer is surgical repair [12]. In most cases of perforation, gastric and duodenal content spills into the peritoneal cavity. This content includes gastric and duodenal secretions, bile, ingested food, and swallowed bacteria. The leakage results in peritonitis, with an increased risk of infection and abscess formation. Subsequent third spacing of fluid in the peritoneal cavity due to perforation and peritonitis leads to inadequate circulatory volume, hypotension, and decreased urine output. In more severe cases, shock may ensue. Abdominal distention as a result of peritonitis and subsequent ileus may interfere with diaphragmatic movement, impairing expansion of lung bases. Eventually, atelectasis develops, which may compromise oxygenation of the blood, particularly in patients with co-existing lung disease [13]. The incidence of mortality due to perforation is $5-10 \%$. Mortality increases up to $50 \%$ if the perforation has been present $>24$ hours. Surgical delay is a wellestablished negative prognostic factor and limiting surgical delay in patients with perforated peptic ulcers (PPU) seems of paramount importance. In men duodenal ulcers are more common than in females. The male-to-female ratio for duodenal ulcers is about 3:1 [14]. Mortality is influenced by a number of factors which include patients age, sex, site of the ulcer, treatment delay, concurrent disease, pre-operative shock, and type of anesthesia used [15]. A majority of the factors are interrelated, and for instance, the treatment delay is likely to increase the mortality rate. Despite a lot of evidence in the literature, the knowledge regarding factors influencing the mortality that occurs after peptic ulcer perforation is limited 14 , 16]. The purpose of this study is to find the factors that influence the mortality and morbidity among operated cases of duodenal ulcer perforation. There are multiple numbers of factors affecting the mortality and morbidity which would be dealt with in this study $[15,14,16]$.

\section{OBJECTIVES}

To find out the post-operative outcome (Mortality and Morbidity) and to correlate the outcome with the prognostic factors.

\section{Inclusion Criteria}

All non-malignant and non-traumatic duodenal ulcer perforation cases above the age of 20 years were included in the study.

\section{Exclusion Criteria}

Traumatic perforation and perforated malignant ulcers were excluded from the study.

\section{MeTHODS}

The following data were recorded in the data sheet such as age, sex, previous history of PUD, nonsteroidal anti-inflammatory drugs (NSAID) intake, duration of symptoms, time lapsed to start treatment, degree of dehydration, hemodynamic status on admission, co morbidities and the amount of peritoneal contamination at operation. Postoperatively the patients were kept under follow up for one month to see the complications like wound infection, burst abdomen, pulmonary atelectasis, pneumonia etc. All the postoperative follow up information were also recorded in the data sheet. A total of 315 cases of Perforated PUD were enrolled in the study over 05 years. All patients underwent laparotomy. Among them 15 cases were found to have perforation in the antral part of the stomach and treated by excision and repair with sending the excised tissue for histopathological evaluation and in reaming 300 cases the perforation was found in the anterior wall of the first part of the duodenum. The patients were treated with perforation closure with pedicled omental patch repair. All the patients were 


\section{ReSUlT}

Table-1: Demographic characteristics of the study population $(n=300)$

\begin{tabular}{|l|l|l|}
\hline \multicolumn{2}{|l|}{ Frequency } & Percentage \\
\hline Age group & 72 & $24.0 \%$ \\
\hline $20-39$ yrs & 174 & $58.0 \%$ \\
\hline $40-59$ yrs & 39 & $13.0 \%$ \\
\hline $60-69$ yrs & 15 & $05.0 \%$ \\
\hline 70 and above & 279 & $93.0 \%$ \\
\hline Sex & $07.0 \%$ \\
\hline Male & 21 & $77.0 \%$ \\
\hline Female & 231 & $23.0 \%$ \\
\hline Socio-economic status \\
\hline Low & 69 & $80.0 \%$ \\
\hline Middle & $20.0 \%$ \\
\hline Residence & 240 & \\
\hline Rural & 60 & \\
\hline Urban &
\end{tabular}

A total of 300 cases of Perforated PUD were enrolled to study the post operative outcome which include the operative mortality, wound infection, burst abdomen and different forms of post operative chest complications. Both male and female patients of all age group ranging from 20 years to $>80$ years were included in this study. Among them 279 (93\%) were male and only $21(07 \%)$ were female. Most of the patients were from rural areas $240(80.0 \%)$ and from low socioeconomic $231(77.0 \%)$ group.

Table-2: Correlation between mortality and different prognostic factors

\begin{tabular}{|l|l|l|l|}
\hline \multicolumn{5}{|l|}{ Patients $(\mathbf{n = 3 0 0})$} & Number of death $(\mathbf{n}=33)$ & Mortality rate \\
\hline Age group & 72 & 03 & $04.1 \%$ \\
\hline $20-39$ yrs & 174 & 18 & $10.3 \%$ \\
\hline $40-59$ yrs & 174 & 05 & $12.8 \%$ \\
\hline $60-69$ yrs & 39 & 07 & $46.6 \%$ \\
\hline 70 and above & 15 & 15 & $35.7 \%$ \\
\hline H/O Concurrent Illness & 42 & 00 & $00.0 \%$ \\
\hline DM & 12 & 06 & $28.6 \%$ \\
\hline HTN & 21 & 1 & $33.3 \%$ \\
\hline COPD & 03 & 12 & $5.4 \%$ \\
\hline IHD & 222 & 08 & $04.7 \%$ \\
\hline Absent & 170 & 05 & $06.7 \%$ \\
\hline Duration of perforation & 09 & $30.0 \%$ \\
\hline$<2$ days & 75 & 11 & $44.0 \%$ \\
\hline 03 days & 30 & 02 & $03.5 \%$ \\
\hline 04 days & 25 & 15 & $23.8 \%$ \\
\hline 05 Days & 57 & 13 & $48.1 \%$ \\
\hline Shock & 63 & 03 & $01.9 \%$ \\
\hline Mild & 27 &
\end{tabular}

In this study, operative mortality was 33 $(11.0 \%)$. It was observed that mortality was increased with some co morbid conditions like old age (>70 yrs), concomitant illness like DM, COPD, IHD, delay to start treatment and presence of significant shock and dehydration. Over the age of 70 years the mortality was as high as $46.6 \%$ whereas in the early age group (60-69 yrs) mortality was only $12.8 \%$. 42 patients were diabetic and operative mortality was $35.7 \%$. Among 21 COPD patients mortality was $28.6 \%$. Whereas only $5.4 \%$ mortality was found in the patients without co morbidities. It was observed that Preoperative delay significantly increases the mortality. In case of pre operative delay of >4days, mortality was as high as $44.0 \%$ whereas that of <2days mortality was significantly less $(04.7 \%)$. 
Mohibul Hassan et al; Sch J App Med Sci, Jan, 2021; 9(1): 86-91

Table-3: Association between complications and co-morbidities

\begin{tabular}{|l|l|l|l|l|l|}
\hline Complications & DM $(\mathrm{n}=42)$ & COPD $(\mathrm{n}=21)$ & IHD $(\mathrm{n}=03)$ & Absent $(\mathrm{n}=222)$ & Total \\
\hline Mortality & $15(35.7 \%)$ & $06(28.6 \%)$ & $1(33.3 \%)$ & $12(5.4 \%)$ & $33(11 \%)$ \\
\hline Wound infection & $30(71.4 \%)$ & $21(100 \%)$ & $1(33.3 \%)$ & $66(29.7 \%)$ & $117(39 \%)$ \\
\hline Burst abdomen & $09(21.4 \%)$ & $12(57.1 \%)$ & $1(33.3 \%)$ & $21(9.5 \%)$ & $32(10.6 \%)$ \\
\hline Chest Infection & $24(57.1 \%)$ & $18(85.7 \%)$ & $1(33.3 \%)$ & $24(10.8 \%)$ & $67(22.3 \%)$ \\
\hline
\end{tabular}

Among the 300 study populations, 78 have got one or more co morbidities. Among them 42 were diabetic, 12 were hypertensive, 21 patients has got COPD and 03 patients had IHD. Among the diabetic patients, mortality rate was $35.7 \%$ (15).In COPD patients; mortality was $28.6 \%$ (06). In non co morbid patients (222), mortality was only $5.4 \%$ (12). Wound infection rate in the diabetic patients was $71.4 \%$, in
COPD patients was $100 \%$. $21.4 \%$ patient developed burst abdomen in the diabetic group. Burst abdomen rate in COPD patients was more that is $57.1 \%$ (12) but in non co morbid patients burst abdomen rate was only $9.5 \%$. Chest infection rate was also high $(57.1 \%)$ in diabetic and COPD patients $85.7 \%$ and that in the non co morbid patients was only $10.8 \%$.

Table-4: Association between complications and Presence of shock

\begin{tabular}{|l|l|l|l|l|l|}
\hline Complications & Presence of shock \\
\hline & Mild $(\mathrm{n}=57)$ & Moderate $(\mathrm{n}=63)$ & Severe $(\mathrm{n}=27)$ & Absent $(\mathrm{n}=153)$ & Total \\
\hline Mortality & $02(3.5 \%)$ & $15(23.8 \%)$ & $13(48.1 \%)$ & $03(01.9 \%)$ & $33(11 \%)$ \\
\hline Wound infection & $24(42.1 \%)$ & $31(49.2 \%)$ & $14(51.9 \%)$ & $51(33.3 \%)$ & $120(40 \%)$ \\
\hline Burst abdomen & $05(8.8 \%)$ & $20(31.7 \%)$ & $09(33.3 \%)$ & $11(7.1 \%)$ & $45(15 \%)$ \\
\hline Chest Infection & $13(22.8 \%)$ & $17(27.0 \%)$ & $09(33.3 \%)$ & $30(19.6 \%)$ & $69(23 \%)$ \\
\hline
\end{tabular}

Degree of shock and dehydration has got significant effect on post operative outcome even we had resuscitated every patient before operation. Severe shock $(\mathrm{n}=27)$ was associated with $48.1 \%$ mortality, 51.9 $\%$ wound infection, $33.3 \%$ burst abdomen and $33.3 \%$ Chest Infection. In patients with mild to moderate shock $(\mathrm{n}=63)$ mortality was found $23.8 \%$, Wound infection was $49.2 \%$, Burst abdomen was $31.7 \%$ and Chest Infection was $27.0 \%$. The patients those who were presented early with mild shock $(n=57)$ or no significant hemodynamic abnormality $(n=153)$, post operative outcome of these patients were Mortality: $3.5 \%$ and $01.9 \%$ respectively, Wound infection: $42.1 \%$ and $33.3 \%$, Burst abdomen: $8.8 \%$ and $7.1 \%$ and Chest Infection: $22.8 \%$ and $19.6 \%$ respectively.

\section{DiscUSSION}

A total of 300 cases of Perforated PUD were enrolled to study the post operative outcome which include the operative mortality, wound infection, burst abdomen and different forms of post operative chest complications. Both male and female patients of all age group ranging from 20 years to $>80$ years were included in this study. Among them 279 (93\%) were male and only $21(07 \%)$ were female. Most of the patients were from rural areas $240(80.0 \%)$ and from low socioeconomic $231(77.0 \%)$ group. In this study, operative mortality was $33(11.0 \%)$. It was observed that mortality was increased with some co morbid conditions like old age (>70 yrs), concomitant illness like DM, COPD, IHD, delay to start treatment and presence of significant shock and dehydration. Over the age of 70 years the mortality was as high as $46.6 \%$ whereas in the early age group (60-69 yrs) mortality was only $12.8 \% .42$ patients were diabetic and operative mortality was $35.7 \%$. Among 21 COPD patients mortality was $28.6 \%$. Whereas only $5.4 \%$ mortality was found in the patients without co morbidities. It was observed that Preoperative delay significantly increases the mortality. In case of pre operative delay of $>4$ days, mortality was as high as $44.0 \%$ whereas that of <2days mortality was significantly less $(04.7 \%)$. A study by Dellinger RP et al., have shown that Sepsis is frequently present and a leading cause of death in patients with perforated peptic ulcer (PPU). An estimated 30-35\% of patients with PPU have sepsis on arrival at the operating theater, and sepsis is believed to account for $40-50 \%$ of fatalities. Within 30 days of surgery, $>25 \%$ of the patients develop septic shock [7], which carries a mortality rate of 50-60\%. Accordingly, investigation and interventions aimed at preventing, detecting, and treating sepsis in PPU patients may reduce mortality and morbidity. This can be accomplished by systematically assessing for the signs of sepsis and treating according to the principles of the surviving sepsis campaign, including fluid resuscitation, cultures, empirical broad-spectrum antibiotics, and source control [17]. A study conducted in Zambia by K. J. Sondashi et al., and found that, Death was the commonest complication arising postoperatively accounting for $37 \%$. This high mortality rate could be attributed to diagnostic and treatment delay, as well as concomitant underlying medical illness, as noted with high rate of HIV infection in the study group [18]. D. L. Buck et al., shown where a total of 2668 patients were included. Their median age was 70.9 (range $16 \cdot 2$ $104 \cdot 2)$ yearsand 55.4 per cent (1478 of 2668) were female. Some 67.5 per cent of the patients (1800 of 2668) had atleast one of six co-morbid diseases and 45.6 per cent had an American Society of Anesthesiologists fitness grade of III or more. A total of 708 patients $(26 \cdot 5$ per cent) died within 30 days of 
surgery. Every hour of delay from admission to surgery was associated with an adjusted 2.4 per cent decreased probability ofsurvival compared with the previous hour (adjusted RR 1.024, 95 per cent c.i. 1.011 to 1.037) [19]. Delayed operation is recognized as a contributor to adverse outcome in many areas of emergency surgery [20]. The primary cause in general surgery seems to be diagnostic delay [20]. Reasons behind delayed surgery for PPU are sparsely explored, but seem to be associated with out-of-hospital perforation, lack of peritoneal signs, late attendance by the surgeon, attendance by a non senior surgeon and lack of pulse oximetry [21]. Patients with out-of-hospital perforation are often unselected and it may take time to reach the diagnosis [20]. Those with atypical symptoms are often not prioritized, compared with patients with signs of an abdominal emergency. Previous studies have reported the strong negative prognostic impact of delayed surgery for PPU [22]. However, the evidence derives primarily from studies using unadjusted analyses, and with few patients [22], risking bias [23], and no study has assessed surgical delay as a continuous variable. A possible reason for the strong association between delay and adverse outcome could be the increased risk of developing severe sepsis. Longstanding perforation is associated with peritoneal contamination, positive peritoneal cultures, septic complications and development of postoperative abscesses [24]. Among the 300 study populations, 78 have got one or more co morbidities. Among them 42 were diabetic, 12 were hypertensive, 21 patients has got COPD and 03 patients had IHD. Among the diabetic patients, mortality rate was $35.7 \%$ (15). In COPD patients; mortality was $28.6 \%$ (06). In non co morbid patients (222), mortality was only $5.4 \%$ (12). Wound infection rate in the diabetic patients was $71.4 \%$, in COPD patients was $100 \%$. $21.4 \%$ patient developed burst abdomen in the diabetic group. Burst abdomen rate in COPD patients was more that is $57.1 \%$ (12) but in non co morbid patients burst abdomen rate was only 9.5\%. Chest infection rate was also high $(57.1 \%)$ in diabetic and COPD patients $85.7 \%$ and that in the non co morbid patients was only $10.8 \%$. S Senthil Arumugam 1 et al., in Tamilnadu conducted a study on 50 cases of duodenal perforation. The patients were treated with perforation closure with live omental patch repair after initial resuscitation. They had shown that the patients aged $>60$ years and associated co morbid illness had the highest rate of wound infection. 17 patients had associated comorbid illness. Of the 8 patients who had wound infection, 6 patients had associated comorbid illness and $50 \%$ of them were above 50 years of age. Electrolyte imbalance included hyponatremia in $21 \%$ of patients, hypokalemia in $19 \%$ of patients, and elevated serum creatinine in $18 \%$ of patients. Mortality rate was $12 \%$, of which 3 patients were treated with $\mathrm{B} / \mathrm{L}$ flank drain because of the very poor general condition of the patient at the time of admission, and all these patients were above the 60 years age group; of the operated patients, $6 \%$ mortality is present, and in these patients, there were associated comorbid illness and delay in presentation and amount of peritoneal contamination were all significantly present [25]. Degree of shock and dehydration has got significant effect on post operative outcome even we had resuscitated every patient before operation. Severe shock $(\mathrm{n}=27)$ was associated with $48.1 \%$ mortality, $51.9 \%$ wound infection, $33.3 \%$ burst abdomen and $33.3 \%$ Chest Infection. In patients with mild to moderate shock $(n=63)$ mortality was found $23.8 \%$, Wound infection was $49.2 \%$, Burst abdomen was $31.7 \%$ and Chest Infection was $27.0 \%$. The patients those who were presented early with mild shock $(n=57)$ or no significant hemodynamic abnormality $(n=153)$, post operative outcome of these patients were Mortality: $3.5 \%$ and $01.9 \%$ respectively, Wound infection: $42.1 \%$ and $33.3 \%$, Burst abdomen: $8.8 \%$ and $7.1 \%$ and Chest Infection: $22.8 \%$ and $19.6 \%$ respectively. Mortality rates for peptic ulcers have remained stable over time in Europe [26] and were reported to be $10-30 \%$ in a 2011 systematic review [27]. However, mortality rates differ substantially between reports (ranging from $3 \%$ to $30 \%$ ), mainly because of geographical variations in causes and patient inclusion, but also differences in method of data collection. Administrative data sources, such as the US National Inpatient Sample [28] and Health Insurance Claims Registry in Korea [29] report low mortality rates (around 3\%). For the USA, such low mortality in administrative datasets contrasts with rates in other reports (mortality of 15\%) from the same country [30]. In prospective, nationwide data collection, such as the Danish Clinical Register of Emergency Surgery, mortality is reported to be as high as $28 \%$ Thus, in addition to geographical variation (Figure-1), method of data capture must be considered carefully when mortality rates are compared [31].

\section{CONCLUSION}

Perforated PUD is acute surgical emergency. Delay in surgery is associated with increased mortality and morbidity. Old age with limited physiological reserve eg. Reduced Cardiorespiratory reserve, DM, IHD, COPD, Heart failure etc is also associated with increased mortality and morbidity. Super added complication like sepsis and septicemia is important factor of bad outcome of surgery.

\section{Source of funding: Self.}

Conflict of interest: None declared.

\section{REFERENCES}

1. Bertleff MJ, Lange JF. Perforated peptic ulcer disease: a review of history and treatment. Digestive surgery. 2010; 27(3):161-169.

2. Møller MH, Adamsen S, Wøjdemann M, Møller AM. Perforated peptic ulcer: how to improve outcome? Scandinavian journal of gastroenterology. 2009; 44(1):15-22.

3. Møller MH, Shah K, Bendix J, Jensen AG, Zimmermann-Nielsen E, Adamsen S, Møller AM. Risk factors in patients surgically treated for peptic 
ulcer perforation. Scandinavian journal of gastroenterology. 2009 Jan 1;44(2):145-52.

4. Mäkelä JT, Kiviniemi H, Ohtonen P, Laitinen SO. Factors that predict morbidity and mortality in patients with perforated peptic ulcers. The European journal of surgery. 2002 Nov 1;168(8-9):446-51.

5. Svanes C. Trends in Perforated Peptic Ulcer: Incidence, Aetiology, Treatment and Prognosis. World J Surg. 2000; 24:277-83.

6. Slade Howell H. When Repair is Enough for Perforated Duodenal Ulcer. Contemporary Surgery. 2008; 64(11): 521-4.

7. Svanes C, Lie RT, Svanes K, Lie SA, Søreide O. Adverse effects of delayed treatment for perforated peptic ulcer. Annals of surgery. 1994 Aug;220(2):168.

8. Ng EK, Chung SC, Sung JJ. High Prevalence Helicobacter Pylori Infection in Duodenal Perforations Not Caused by Non-Steroidal AntiInflammatory Drugs. Br J Surg. 1996; 83: 1779 -81 .

9. Sah DN. Predicting Outcome in Perforated Peptic Ulcer from Tertiary Referral Center of Nepal. ARC Journal of Surgery. 2019; 5(1):9-14.

10. Di Saverio S, Bassi M, Smerieri N, Masetti M, Ferrara F, Fabbri C, Ansaloni L, Ghersi S, Serenari M, Coccolini F, Naidoo N. Diagnosis and treatment of perforated or bleeding peptic ulcers: 2013 WSES position paper. World Journal of Emergency Surgery. 2014 Dec 1;9(1):45.

11. Søreide K, Thorsen K, Harrison EM, Bingener J, Møller MH, Ohene-Yeboah M, Søreide JA. Perforated peptic ulcer. The Lancet. 2015 Sep 26;386(10000):1288-98.

12. Chang H, Choi W. Nonoperative Treatment of Perforated Duodenal Ulcer: A Case Report and Review of the Literature. Journal of Emergency Critical Care in Medicine. 2007 Dec 1;168:18.

13. Sharma SS. A prospective cohort study of postoperative complications in the management of perforated peptic ulcers. BMC Surg. 2006; 8:23-25.

14. Buck DL, Vester-Andersen M, Møller MH. Surgical delay is a critical determinant of survival in perforated peptic ulcer. $\mathrm{Br}$ J Surg. 2013; 100(8):1045-49.

15. Lehours P, Yiilmaz O. Epidemiology of helicobacter pylori infection. Helicobacter. 2007; 12:1-3.

16. Stabins SJ, Rochester NY. The aftermath of perforated duodenal ulcer. Surgery. 1953; 34:614-20.

17. Campaign SS, Dellinger RP, Levy MM, Rhodes A, Annane D, Gerlach H, Opal SM, Sevransky JE, Sprung CL, Douglas IS, Jaeschke R. International guidelines for management of severe sepsis and septic shock: 2012. Crit Care Med. 2013 Feb;41(2):580-637.

18. Sondashi KJ, Odimba BF, Kelly P. A cross-sectional study on factors associated with perforated peptic ulcer disease in adults presenting to UTH, Lusaka. Medical Journal of Zambia. 2011;38(2):15-22.

19. Buck DL, Vester- Andersen M, Møller MH, Danish Clinical Register of Emergency Surgery. Surgical delay is a critical determinant of survival in perforated peptic ulcer. British Journal of Surgery. 2013 Jul;100(8):1045-9.

20. North JB, Blackford FJ, Wall D, Allen J, Faint S, Ware RS, Rey- Conde T. Analysis of the causes and effects of delay before diagnosis using surgical mortality data. British journal of surgery. 2013 Feb;100(3):419-25.

21. Møller MH, Nørgård BM, Mehnert F, Bendix J, Nielsen AS, Nakano A, Adamsen S, Thomsen RW. Preoperative delay in patients with peptic ulcer perforation: a clinical audit from the Danish National Indicator Project. Ugeskrift for laeger. 2009 Nov 1;171(49):3605-10.

22. Møller MH, Adamsen S, Thomsen RW, Møller AM. Preoperative prognostic factors for mortality in peptic ulcer perforation - a systematic review. Scand J Gastroenterol. 2010; 45: 785-805.

23. Guyatt GH, Oxman AD, Vist G, Kunz R, Brozek J, Alonso-Coello P, Montori V, Akl EA, Djulbegovic B, Falck-Ytter Y, Norris SL. GRADE guidelines: 4. Rating the quality of evidence-study limitations (risk of bias). Journal of clinical epidemiology. 2011 Apr 1;64(4):407-15.

24. Fong IW. Septic complications of perforated peptic ulcer. Can J Surg. 1983; 26: 370-372.

25. Senthil-Arumugam S, Parthiban G, Ramprasad A, Heber A. A Study of Duodenal Ulcer Perforation Post-operative Outcome in A Tertiary Center. International Journal of Scientific Study. October 2018; 6(70). DOI: 10.17354/ijss/2018/8

26. Sonnenberg A. Time trends of ulcer mortality in Europe. Gastroenterology. 2007; 132: 2320-27.

27. Lau JY, Sung J, Hill C, Henderson C, Howden CW, Metz DC. Systematic review of the epidemiology of complicated peptic ulcer disease: incidence, recurrence, risk factors and mortality. Digestion. 2011; 84:102-13.

28. Wilhelmsen M, Moller MH, Rosenstock S. Surgical complications after open and laparoscopic surgery for perforated peptic ulcer in a nationwide cohort. $\mathrm{Br}$ J Surg. 2015; 102: 382-87.

29. Bae S, Shim KN, Kim N, Kang JM, Kim DS, Kim KM, Cho YK, Jung SW. Incidence and short-term mortality from perforated peptic ulcer in Korea: a population-based study. Journal of epidemiology. 2012 Nov 5;22(6):508-16.

30. Wang YR, Richter JE, Dempsey DT. Trends and outcomes of hospitalizations for peptic ulcer disease in the United States, 1993 to 2006. Ann Surg. 2010; 251: 51-58.

31. Buck DL, Møller MH, Danish Clinical Register of Emergency Surgery. Influence of body mass index on mortality after surgery for perforated peptic ulcer. British Journal of Surgery. 2014 Jul;101(8):993-9. 